\title{
Port site Metastasis in Prostate Cancer after Laparoscopic Radical Prostatectomy: A Case Report
}

\author{
Guoqiang ${ }^{1}$ Qin, Ge Zou*1 and Weide Zhong*2 \\ ${ }^{1}$ Department of Urology, Central Hospital of Panyu District, China \\ ${ }^{2}$ Department of Urology, Guangdong Key Laboratory of Clinical Molecular Medicine and Diagnostics, China
}

Received: June 4, 2018; Published: June 11, 2018

*Corresponding author: Ge Zou, Central Hospital of Panyu District, 8 Fuyu Road, Panyu, Guangzhou, Guangdong,511400, P.R. China

Weide Zhong, Department of Urology, Guangdong Key Laboratory of Clinical Molecular Medicine and Diagnostics, Guangzhou First People's Hospital, the Second Affiliated Hospital of South China University of Technology'1 Panfu Road, Yuexiu, Guangzhou, Guangdong 510180, P.R. China

Abstract

Background: The port site metastasis in prostate cancer(PCa) after laparoscopic radical prostatectomy(LRP) is an uncommon metastatic pattern of PCa.

Case presentation: We described an 81-year-old male patient who was admitted because of dysuria. Physical examination and imaging study indicated that the port site metastasis in PCa after LRP, and laboratory analysis revealed elevated prostate specific antigen (PSA). The diagnosis was established after tumor resection, and then confirmed by pathology according to immunohistochemical stain. Androgen deprivation therapy(ADT) was given.

Conclusions: Our present case indicated that the port site metastasis in PCa after LRP is rare. Tumor resection is the first choice of treatment and ADT should be performed in case of highly PSA level.

Keywords: Prostate cancer; Metastasis; Laparoscopic radical prostatectomy

Abbreviations: PC: Prostate cancer; PSA: prostate specific antigen; RRP: Radical retropubic prostatectomy; LRP: laparoscopic radical prostatectomy; ADT: Androgen deprivation therapy

\section{Background}

Prostate cancer(PCa) is the first most commonly diagnosed malignancy in men in the Western world and the third leading cause of cancer-related deaths among men [1]. Because of the popularity of prostate specific antigen (PSA) screening, more and more men detected PCa early. Radical retropubic prostatectomy(RRP) is a standard surgical procedure for the treatment of localized PCa. At present, laparoscopic radical prostatectomy(LRP) has been very commend, the operation effect is equivalent to RRP and the complications of the operation are not significantly increased [2]. In 2014, a patient admitted in our hospital who was found the port site metastasis in PCa after LRP. We reviewed the literatures and found that there were few cases of the port site metastasis in PCa after LRP. Therefore, we reported the case and follow-up results.

\section{Case Presentation}

An 81-year-old male patient with 3-year history of dysuria came to our hospital, who was underwent LRP in the Second Affiliated Hospital of SUN YAT-SEN University in 2005. Unfortunately, the patient was missing follow-up after operation. Physical examination revealed that an egg size, fixed tumor on the right side of the abdomen, and nothing was detected in digit rectal exam. The ultrasonography found that the tumor was located in the subcutaneous tissue with abundant blood supply, and SPECT showed bone metastasis in lumbar spine. Laboratory data suggested an elevated PSA level of $57.92 \mathrm{ng} / \mathrm{ml}$. For further investigation, computed tomography(CT) was taken. The CT showed that the tumor with uneven density and reinforcement on contrast-enhanced scan was located in the abdominal muscle layer, which indicated the possibility of metastatic tumor (Figure 1). So, we did a tumor resection, and for relieving lower urinary tract symptoms(LUTS) transurethral resection of bladder neck(TURBN) was performed at the same time. The pathology (Figure 2) suggested that the tumor was adenocarcinoma of the prostate glan.

\section{port site metastasis}

We suggested that the patient undergo androgen deprivation therapy(ADT) after operation because of the highly PSA level, but 
the final decision in patient was to oral bicalutamide. The PSA level decreased from the initial $57.92 \mathrm{ng} / \mathrm{ml}$ to a minimum of $1.6 \mathrm{ng} /$ $\mathrm{ml}$, and then began to up to $8.8 \mathrm{ng} / \mathrm{ml}$ during that period, so we increased the subcutaneous injection of goserelin release agent based on oral bicalutamide until the PSA level finally decreased to $0.18 \mathrm{ng} / \mathrm{ml}$.

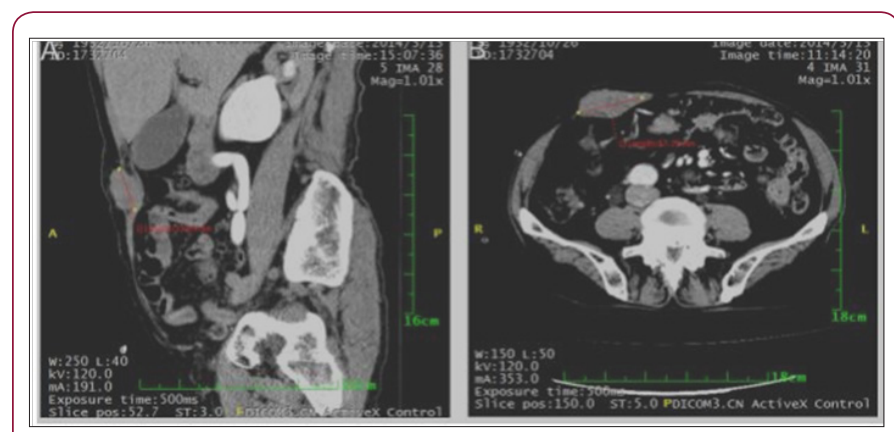

Figure 1: computed tomography(CT).

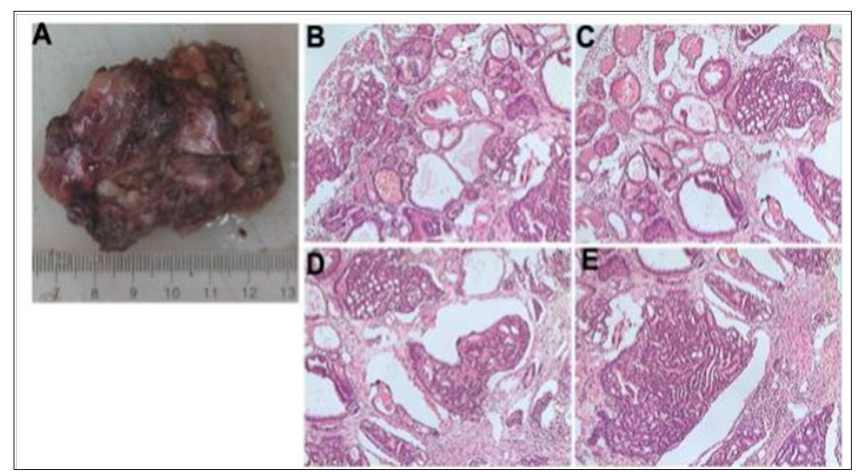

Figure 2: Immunohistochemical Stain.

\section{Discussion}

PCa is a common malignancy in men. RRP is the standard treatment for localized PCa because of better tumor-free survival and metastasis-free survival. Researches showed the complications in the perioperative and the rate of tumor control in LRP were equivalent to RRP [3-5]. The most common metastatic sites of PCa were the bone, distant lymph nodes [6], occasional reports of liver [7] and renal [8] metastasis. The metastasis of PCa was closely correlated with preoperative PSA level, tumor pathological grade and clinical stage, involving a large number of genes, pathways, etc., However, the specific mechanisms involved were still unclear. To the best of our knowledge from literature searches, there were very few literatures that reported on the port site metastasis in PCa after LRP. The first reporters were El-Tabey NA and Shoma AM [9], and after they had some similar reports [10-12].

These reports suggested that the port site metastasis in PCa was extremely rare, but unfortunately usually associated with poor prognosis and endoscopic surgical techniques. We suggest that a systemic assessment should be carried out to assess the presence of local clinical recurrence or distant metastasis, such as bone metastasis, liver metastasis, lung metastasis if highly suspected the port site metastasis in PCa after LRP. The best way is to remove the tumor, and ADT after surgery is feasible if there are some evidences of local clinical recurrence or distant metastasis. If necessary, combined with local radiotherapy.

\section{Conclusion}

The port site metastasis in PCa after LRP is rare. Tumor resection is the first choice of treatment When it happens, and If local clinical recurrence or distant metastasis occurs at the same time, ADT or radiation therapy should be performed.

\section{References}

1. Siegel RL, Miller KD, Jemal A(2017) Cancer Statistics. CA Cancer J Clin 67(1): 7-30.

2. Magheli A, Busch J, Leva N, Schrader M, Deger S, Miller K, et al. (2014) Comparison of surgical technique (open vs. laparoscopic) on pathological and long term functional outcomesfollowing radical prostatectomy.BMC Urol7:14-18.

3. Hruza M, Bermejo JL, Flinspach B, Schulze M, Teber D, et al. (2013) Longterm oncological outcomes after laparoscopic radical prostatectomy.BJU Int111(2):271-280.

4. Novara G, Ficarra V, Mocellin S, Ahlering TE, Carroll PR, et al. (2012) Systematic review and meta-analysis of studies reporting oncologic outcome after robot-assisted radical prostatectomy.Eur Urol62(3):382404.

5. Ficarra V, Novara G, Artibani W, Cestari A, Galfano A, et al. (2009) Retropubic, laparoscopic, and robot-assisted radical prostatectomy: a systematic review and cumulative analysis of comparative studies.Eur Urol55(5):1037-1063.

6. Gandaglia G, Abdollah F, Schiffmann J, Trudeau V, Shariat SF, et al. (2014) Distribution of metastatic sites in patients with prostate cancer: apopulation-based analysis. Prostate74:210-216.

7. Hisashi Hidaka, Makoto Ohbu, Takahide Nakazawa, Takaaki Matsumoto, Akitaka Shibuya, et al. (2015) Peliosis hepatis disseminated rapidly throughout the liver in a patient with prostate cancer: a case report. Journal of Medical Case Reports 9: 194.

8. Chao Chen, Huadong He, Zhijian Yu, Yuansong Qiu, Xuliang Wang (2016) Renal and retroperitoneal metastasis from prostate adenocarcinoma: a case report.World Journal of Surgical Oncology 14:74.

9. ElTabey NA, Shoma AM (2005) Port site metastases after robot-assisted laparoscopic radical cystectomy.Urology66(5):1110.

10. Savage SJ, Wingo MS, Hooer HB, Smith MT, Keane TE (2007) Pathologically confirmed port site metastasis after laparoscopic radical prostatectomy: case report and literature review.Urology70(6):1222, e9-11.

11. Shin YS, Doo AR, Kim MK, Jeong YB, Kim HJ (2012) First case of perit toneal seeding of prostate cancer during robot-assisted laparoscopic radical prostatectomy.Can J Urol19(3):6303-6305.

12. Acar O, Esen T, Bavbek S, Peker O, Musaoğlu A (2014) Port site and peritoneal metastases after robot-assisted radical prostatectomy.Int J Surg Case Rep5(3):131-134. 
(C) (i) This work is licensed under Creative

Submission Link: https://biomedres.us/submit-manuscript.php

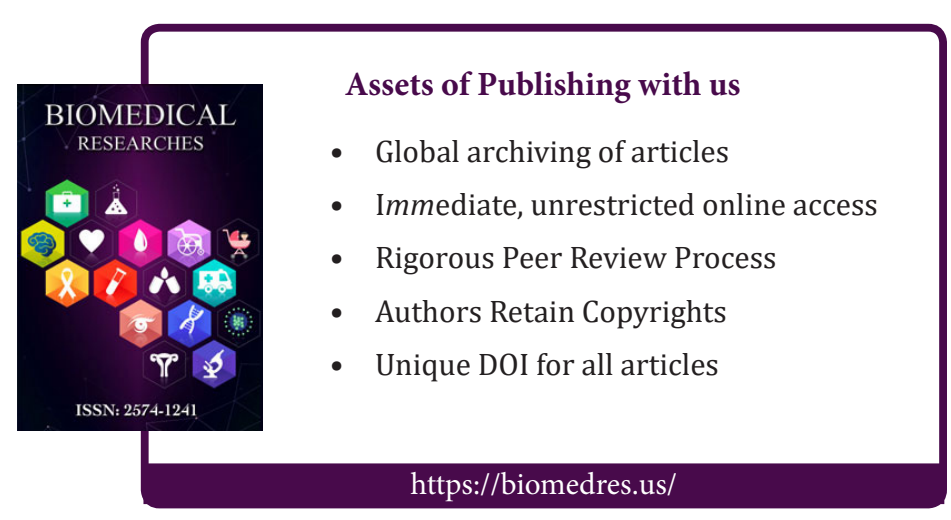

